\title{
The Effect of Hydrated Sodium Calcium Aluminosilicate on Fatty Liver and the Composition of the Intestinal Microbiota in Overfed Landes Geese
}

\section{-Author(s)}

\section{Tang J'," \\ Fang Q" \\ Lu M ${ }^{\text {III }}$ \\ Shao Riv \\ Shen J" \\ Lu L" \\ Niu $D^{\prime}$}

College of Animal Sciences, Zhejiang University, Hangzhou 310058, P. R. China.

" Institute of Animal Husbandry and Veterinary Science, Zhejiang Academy of Agricultural Sciences, Hangzhou 310021, P. R. China.

III Kaier Environmental Technology Co., Ltd. of Hangzhou, Xiaoshan, Hangzhou 311200, China.

Iv Rongyao goose industry Co., Ltd of Changxing, Huzhou 313100, China.

\section{Mail Address}

Corresponding author e-mail address Dong Niu \& Lizhi Lu

Zhejiang University - College of Animal Sciences - 866 Yuhangtang Road,

Hangzhou, Zhejiang Province, 310058, P.

R. China.

Email: dniu@zju.edu.cn lulizhibox@163.com

\section{- Keywords}

Landes geese; microbial diversity; overfeeding; hydrated sodium calcium aluminosilicate; high-throughput sequencing.

\section{ABSTRACT}

Goose fatty liver is a delicious food product and the overfeeding will cause the abnormal physiology of the geese. The objective of this study was to investigate the effect of supplementation with hydrated sodium calcium aluminosilicate (HSCAS) on the fatty liver, ileal and cecal microbiota of Landes geese during overfeeding. Sixty 70-day-old Landes geese (body weight $=3.0 \pm 0.05 \mathrm{~kg}$ ) were randomly divided into three groups, two of which were overfed with whole corn supplemented with or without HSCAS for 20 days when the fatty liver reaches to the maximum size and the negative control group was ad libitum access to the corn basal diet. The intestinal contents of the ileum and cecum from three geese per group were used for high-throughput sequencing. As a result of this study, the HSCAS-treatment led to an increase in relative liver weight $(p<0.05)$ of geese compared with the overfeeding control group. The richness and diversity of the bacterial communities decreased in the ileum and ceca after overfeeding. Overfeeding increased the relative abundance of Firmicutes, especially Lactobacillus, in ileal samples. HSCAS supplementation increased the relative abundance of Lactobacillus, and decreased the relative abundance of Actinobacillus in the ileum and the relative abundance of Erysipelotrichi, Bacteroides and Escherichia in the ceca. Bacterial richness indicators were also increased in samples from ileum and ceca after HSCAS supplementation. In conclusion, dietary HSCAS supplementation promoted liver performance in overfed Landes geese. HSCAS treatment had a beneficial effect on the intestinal microbiota composition in geese during the overfeeding.

\section{INTRODUCTION}

The goose liver has a high capacity for fat accumulation, and the Landes goose is highly susceptible to hepatic steatosis (Lu et al., 2015). During overfeeding (OF), the birds were fed exclusively with corn, which is rich in carbohydrates (especially starch). This diet induces hepatic steatosis, resulting in storage of fatty acids in the liver and producing foie gras (Davail et al., 2003; Liu et al., 2016). Furthermore, OF has an effect on the bacterial composition of the microbiota in these ducks. This diet has been shown to affect bacterial communities (Vasai et al., 2014a; Vasai et al., 2014b).

The intestinal microbiota has a symbiotic relationship with the host and plays a major role in animal physiology (Vasai et al., 2014b). For example, intestinal bacteria can affect the gut morphology and nutrition and stimulate the immune response to protect against pathogens (Leser \& Molbak, 2009). Culture-dependent methods have shown that facultatively anaerobic groups of bacteria are the predominant flora of the small intestine, whereas obligate anaerobes comprise nearly the entire microbial population of the cecum (Torok et al., 2011). A study 
of two parental genetic types of ducks (Pekin and Muscovy) showed that the ileal and cecal microbiota were mainly composed of Firmicutes and Bacteroidetes (Vasai et al., 2014a). Different stresses experienced by animals, such as a change in diet, can modify the composition of the microbiota (Serino et al., 2012).

Mycotoxins can be harmful to hosts. Mycotoxincontaminated feed may lead to consequences such as poor growth performance and meat quality, low nutrient digestibility, and increased disease incidence due to immunosuppression, all of which may result in economic losses (Swamy et al., 2002; Shang et al., 2016). Aflatoxin (AF) is a mycotoxin that particularly affects poultry and often occurs in naturally contaminated feeds (Aravind et al., 2003; Murugesan et al., 2015). A study with chickens showed that dietary supplementation with hydrated sodium calcium aluminosilicates (HSCAS) was effective in binding AF molecules in the gastrointestinal tract, making them unavailable for adsorption and therefore alleviating aflatoxicosis (Chen et al., 2014). In the poultry industry, the interest in HSCAS is increasing. Because the use of antibiotics in food animals for growth promotion and disease prevention may lead to antibiotic resistance in humans and animals, several countries have restricted the use of antibiotics in livestock feed as a public health measure (Salim et al., 2013). The beneficial effects of dietary HSCAS have been recognized for the last three decades (Tanpong et al., 2017), and it may serve as an alternative to antibiotic growth stimulants without clinical side effects (Gilani et al., 2016). Although their nutritional properties have been widely studied in animals, to date, few reports are available on goose liver changes in response to OF. High-throughput $16 S$ sequencing of major intestinal bacteria may help explain the effects and mechanisms of HSCAS in geese, as no similar studies have been published.

Therefore, the objectives of this study were to determine the effects of an HSCAS supplement in overfed geese by making an inventory of fatty liver and intestinal microbiota in both ileum and ceca, and the effect of overfeeding to know the impact of a food stress on the microbiota was studied using a highthroughput amplicon sequencing.

\section{MATERIAL AND METHODS}

\section{Experimental design}

All experimental procedures involving geese were conducted in accordance with the guidelines set by the Animal Care and Use Committee of Zhejiang University for the care of animals for research purposes. Geese were raised on a farm belonging to the ChangXing Glory Goose Industry Co. Ltd., Huzhou, China.

A total of 60 healthy 70-day-old Landes geese (BW $=3.0 \pm 0.05 \mathrm{~kg}$ ), hatched on the same day and bred under natural light and temperature conditions. They were randomly divided into three groups: 1) ad libitum access to a corn basal diet containing no additives (negative control group, Control I); 2) overfed with a corn basal diet (positive control group, Control II); 3) overfed with a corn basal diet supplemented with $0.3 \%$ HSCAS product (treatment group). Each treatment had four replicate pens with five geese in each pen. The movement of the birds was not restricted before day 0 of overfeeding, but all of the birds were restricted as they were moved to cages for the period of adaptation and OF. A 5-day-long pre-OF period prepared the OF group for formal OF, which lasted $20 \mathrm{~d}$. The OF procedure and diet regimes were implemented as previously described (Lu et al., 2015). Routine husbandry was carried out throughout the experiment.

\section{Tissue sampling}

The geese were sacrificed at day 20 of OF. Five birds from each group were randomly selected for weighting, scalding and de-feathering. Livers and the carcass were weighed. The intestinal content from the ileum and cecum was collected, and the samples were stored at $-20{ }^{\circ} \mathrm{C}$. The intestinal contents of the ileum and cecum from three geese per group were used for high-throughput sequencing, and analyses were performed by Beijing Novogene Biotechnology Co., Ltd (Xie et al., 2016).

\section{DNA extraction, library preparation, and sequencing of 165 rRNA amplicons}

Total DNA from ileal and cecal samples was extracted using the CTAB/SDS method. DNA concentration and purity were monitored on $1 \%$ agarose gels. Based on the concentration, the extracted DNA was diluted to $1 \mathrm{ng} / \mu \mathrm{L}$ using sterile water and stored at $-20^{\circ} \mathrm{C}$ until PCR was performed. Amplicons of the $\mathrm{V} 4$ region of the 16S rRNA of samples were amplified using bacterial primers 515F (GTGCCAGCMGCCGCGGTAA, forward) and 806R (GGACTACNNGGGTATCTAAT, reverse) (Caporaso et al., 2010). All PCR reactions were carried out using Phusion High-Fidelity PCR Master Mix (New England Biolabs).

A mix of the same volume of $1 \times$ loading buffer (containing SYB green) and PCR products was subjected to electrophoresis on a $2 \%$ agarose gel for detection of amplicons. Samples with a bright band between 
Tang J, Fang Q, Lu M, Shao R, Shen J, Lu L, Niu D
The Effect of Hydrated Sodium Calcium

Aluminosilicate on Fatty Liver and the Composition of the Intestinal Microbiota in Overfed Landes Geese
400-450 bp were chosen for further experiments. PCR products were mixed in equidensity ratios. Then, the mixture PCR products was purified using a Qiagen Gel Extraction Kit (Qiagen, Germany) (Nylund et al., 2010).

Sequencing libraries were generated using TruSeq DNA PCR-Free Sample Preparation Kit (Illumina, USA) according to the manufacturer's recommendations, and index codes were added. Library quality was assessed on the Qubit@ 2.0 Fluorometer (Thermo Scientific) and Agilent Bioanalyzer 2100 system. Finally, the library was sequenced on an Illumina HiSeq2500 platform, and 250-bp paired-end reads were generated (Du et al., 2016).

\section{Paired-end read assembly and quality control}

Paired-end reads was assigned to samples based on their unique barcode and truncated by removing the barcodes and primer sequences. Paired-end reads were merged using FLASH (version 1.2.7); the splicing sequences were called raw tags. Quality filtering of the raw tags was performed under specific filtering conditions to obtain high-quality clean tags based on the QIIME (version 1.7.0) quality control process (Bokulich et al., 2013). The tags were compared with a reference database (the GOLD database, http://drive5. com/uchime/uchime_download.html) using a UCHIME algorithm to detect chimeric sequences, and then chimeras were removed (Haas et al., 2011). Finally, effective tags were obtained.

\section{Taxonomic classification and statistical analysis}

Sequences analyses were performed using Uparse software (Uparse version 7.0.1001). Sequences with $\geq$ $97 \%$ similarity were assigned to the same operational taxonomic units (OTUs). Representative sequence for each OTU was screened for further annotation. For each representative sequence, the GreenGene Database (http://greengenes. Ibl.gov/cgi-bin/nph-index. cgi) was used with the Ribosomal Database Project (RDP) Classifier (version 2.2) algorithm to annotate taxonomic information. In order to determine the phylogenetic relationships of different OTUs, and the differences between dominant species in different samples (groups), a multiple sequence alignment was generated using MUSCLE (version 3.8.31). OTU abundances were normalized using a standard of sequence numbers corresponding to the sample with the least sequences. A subsequent analysis of alpha and beta diversities was performed based on this normalized data. Alpha diversity indices, including the observed species, Chao1 (the estimated true species diversity of a sample), Shannon (biodiversity), ACE (coverage of sampling), and Good's coverage indices, were used to analyze the complexity of species diversity in a sample All of these indices were calculated in QIIME (version 1.7.0) and displayed using R software (version 2.15.3). A beta diversity analysis was used to evaluate differences in species complexity between samples, and beta diversity based on both weighted and unweighed Unifrac were calculated in QIIME (version 1.7.0). A $t$-test was performed to determine the statistical significance of differences.

\section{Statistical analyses}

Carcass, liver weight, and relative liver weight data are presented as mean \pm SE. Data were analyzed as a completely randomized design. A comparison of variables was performed using an independent-samples t-test and one-way ANOVA with SPSS v. 20.0 software (SPSS Inc., 2011). Differences among treatment means were determined using Duncan's multiple range test. The results were considered significantly different at $p \leq 0.05$.

\section{RESULTS}

\section{Body and liver parameters}

All geese (except the control group) consumed the diet provided. As shown in Figure 1, HSCAS
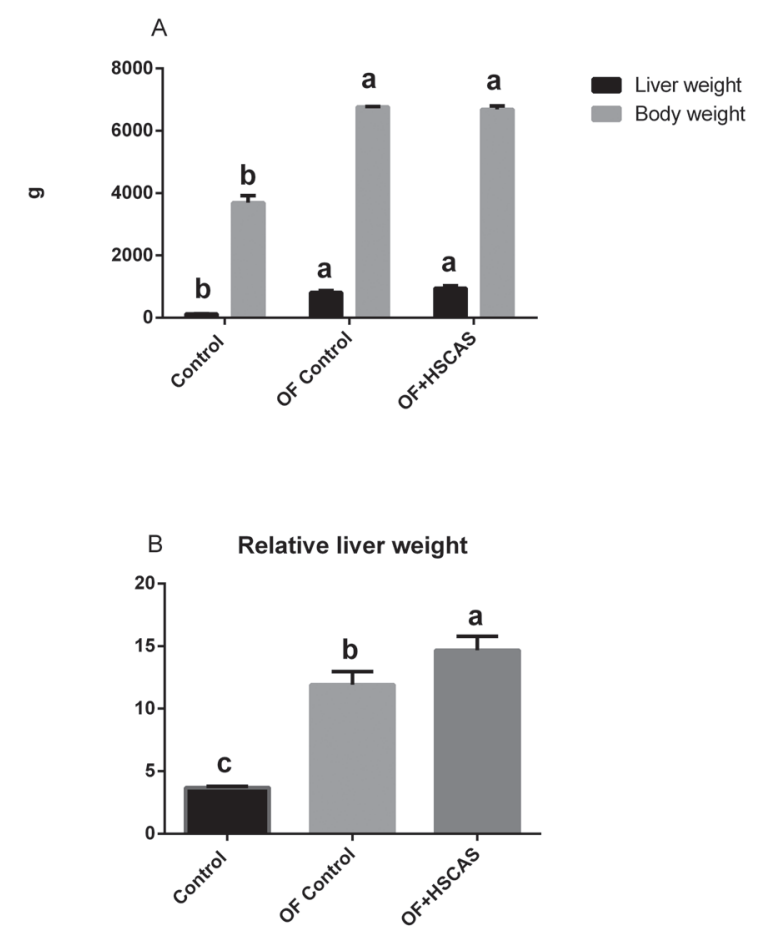

Figure 1 - Effects of HSCAS supplementation on body weight and fatty liver performance of overfed Landes geese. 
Tang J, Fang Q, Lu M, Shao R, Shen J, Lu L, Niu D
The Effect of Hydrated Sodium Calcium

Aluminosilicate on Fatty Liver and the Composition of the Intestinal Microbiota in Overfed Landes Geese treatment led to an increase in liver weight, body weight and liver/body weight compared with the control group $(p<0.05)$. The HSCAS-treated group also had greater liver weight and liver/body weight compared with the overfeeding control group, which improved by $17.35 \%(140 \mathrm{~g})(p>0.05)$ and $2.28 \%$ $(p<0.05)$, respectively. No difference was observed in body weight between the geese fed the overfeeding diet without or with HSCAS.

\section{Bacterial communities in Landes geese}

The microbial diversity in samples was estimated by calculating the number of OTUs. For each ileal sample, the number of OTUs based on a sequence similarity cutoff of 0.05 was $598 \pm 135$, with sample coverage of $99.00 \pm 0.25 \%$. The average number of sequences was 90,544, and eight different phyla were recorded among 131 different taxa. The Chao1, ACE, and Shannon indices were $1048.46 \pm 322.72,1191.99 \pm$ 408.70, and 3.24 \pm 0.27 , respectively (Table 1).

Table 1 - Estimators of diversity during overfeeding (OF) in the ileum and ceca of Landes geese

\begin{tabular}{lcccc}
\hline \multirow{2}{*}{ Item } & \multicolumn{4}{c}{ Group } \\
\cline { 2 - 5 } & Control & OF Control & HSCAS & SEM \\
\hline lleum & \multicolumn{4}{c}{} \\
\hline Number of OTUs & 598 & 478 & 985 & 184.73 \\
Chao1 & 1048.46 & 954.16 & 1778.78 & 322.72 \\
ACE & 1191.99 & 1206.91 & 2197.34 & 408.70 \\
Shannon & 3.24 & 2.80 & 2.43 & 0.27 \\
\hline Ceca & \multicolumn{4}{c}{474} \\
\hline Number of OTUs & 579 & 497 & 47.90 \\
Chao1 & 630.02 & 551.73 & 586.96 & 42.10 \\
ACE & 652.41 & 567.63 & 616.39 & 40.37 \\
Shannon & 5.84 & 4.01 & 4.22 & 0.37 \\
\hline
\end{tabular}

ACE, abundance-based coverage estimator; OTU, operational taxonomic unit; SEM, standard error of the mean.

The majority of the diversity in the ileum before OF was represented by Proteobacteria sequences $(49.4 \%)$. The rest of the sequences were composed of Firmicutes (21.1\%) and Actinobacteria (8.3\%) OTUs. Other phyla such as Bacteroidetes or Fusobacteria represented less than $2 \%$ of the sequences (Figure $2 a)$. At the class level, the microbiota were mainly composed of Gammaproteobacteria (23.69\%) and Alphaproteobacteria (12.16\%) in the phylum Proteobacteria; Clostridia (5.21\%) and Bacilli (14.21\%) in the Firmicutes; and Bacteroidia (1.46\%) in the Bacteroidetes (Figure 2b).

Regarding cecal samples, the number of OTUs based on a sequence similarity cutoff of 0.05 was 579 \pm 132 . The average number of sequences was 64,056 , with 10 phyla represented by 167 different taxa. The
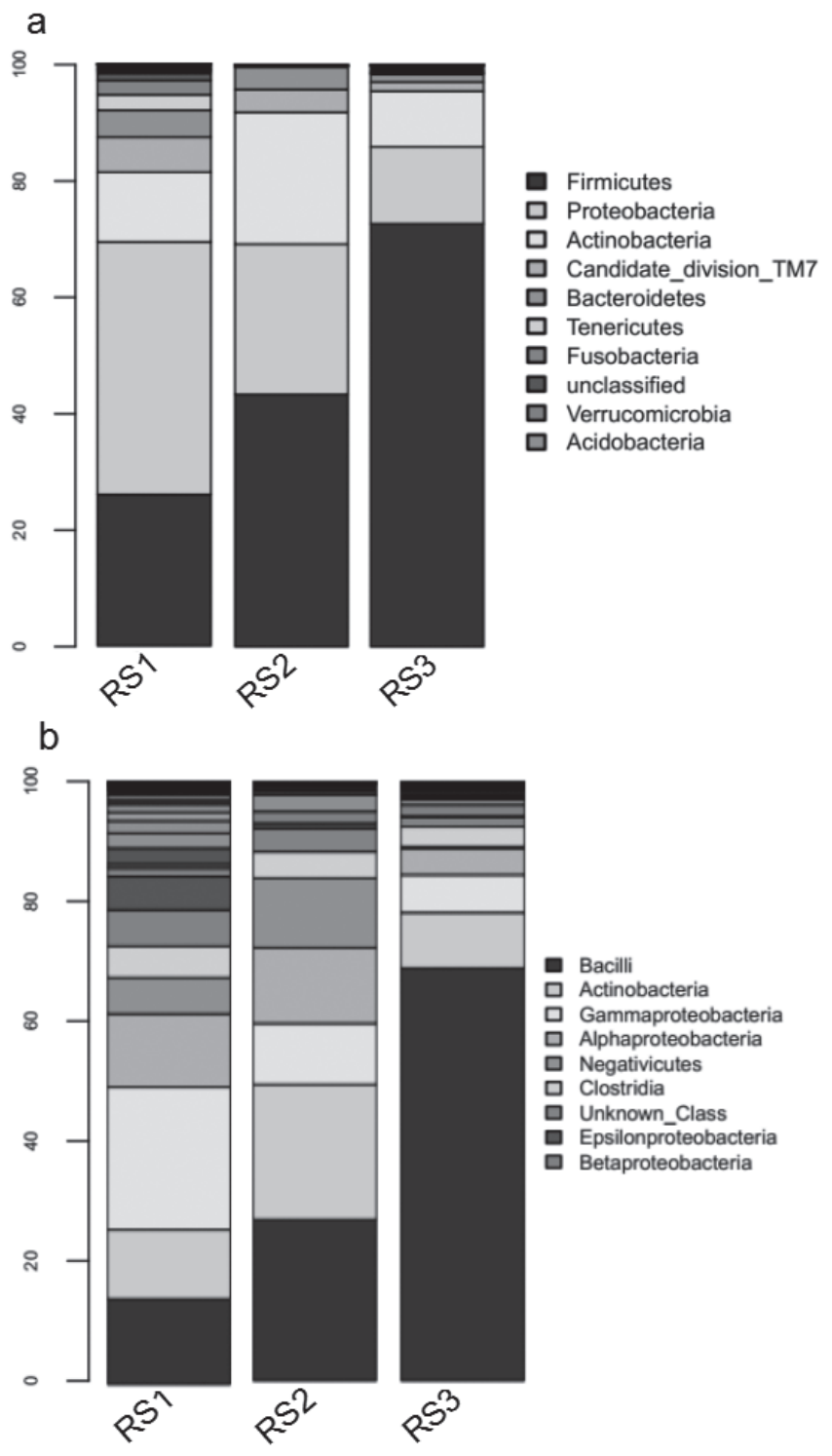

Figure 2 - Percentage contribution of sequences at the phylum (a) and class (b) levels to the total number of sequences in ileum during overfeeding (OF), with or without HSCAS treatment. Non-OF (RS1), OF without HSCAS supplementation (RS2), and OF with HSCAS supplementation (RS3).

Chao1, ACE, and Shannon indices were $630.02 \pm$ $42.10,652.41 \pm 40.37$, and $5.84 \pm 0.37$, respectively (Table 1). The sequences retrieved from the ceca were mainly composed of Bacteroidetes OTUs (38.5\%). Firmicutes sequences amounted to $45.5 \%$ of the total, and Proteobacteria sequences accounted for $6.6 \%$ (Figure 3a). The sequences from two classes, Bacteroidia (38.4\%) of the phylum Bacteroidetes, and Clostridia (34.4\%) from the phylum Firmicutes, were dominant in the ceca (Figure 3b).

\section{Effect of HSCAS on the ileal microbial communities in overfed geese}

In samples from HSCAS-overfed geese, indicators showed increased species richness in the ileum and 

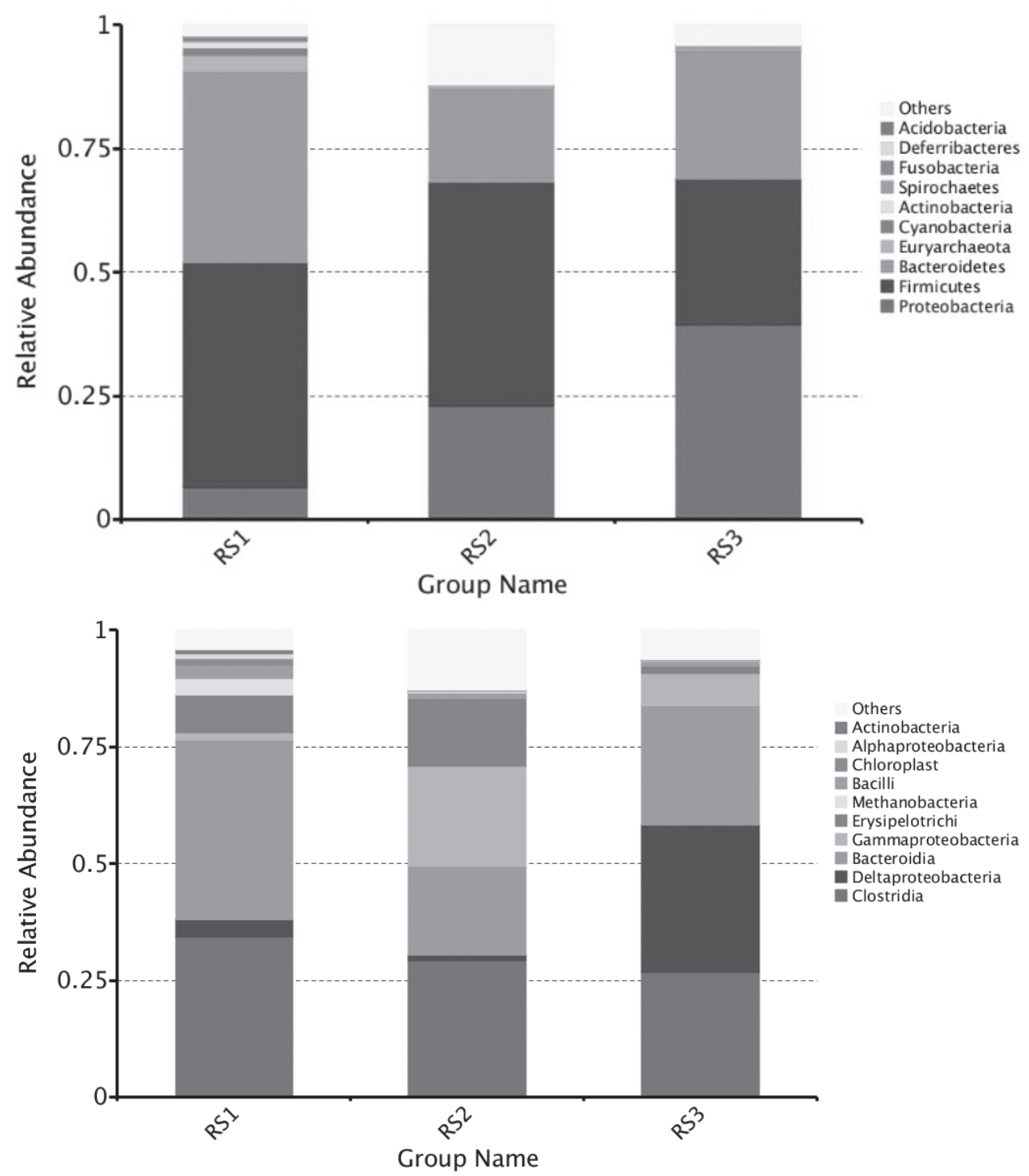

Figure 3 Percentage contribution of sequences at the phylum (a) and class (b) levels to the total number of sequences in cecum during overfeeding (OF), with or without HSCAS treatment. Non-OF (RS1), OF without HSCAS supplementation (RS2), and OF with HSCAS supplementation (RS3).

ceca at end of OF compared to control II. In the ileum, before OF, microbiota were mainly composed of Gammaproteobacteria (23.69\%), followed by Bacilli (14.21\%), Alphaproteobacteria (12.16\%), Actinobacteria (11.52\%), Clostridia (5.21\%), and Bacteroidia (1.46\%), as indicated by the sequencing data (Figure 2a).

At the end of the OF phase, Bacilli were most well represented (26.97\%) among the sequences, followed by Actinobacteria (22.58\%), Gammaproteobacteria (10.22\%), and Alphaproteobacteria (12.64\%), whereas Clostridia represented only $4.34 \%$ of the population and Bacteroidia were almost nonexistent $(0.53 \%)$ (Figure 2b). A statistically significant decrease in Helicobacter and Pseudoalteromonaswas observed in the sequencing results $(p<0.05)$. After
OF, Actinobacteria, including Corynebacterium; Gammaproteobacteria, including Actinobacillus; and Lactobacillus in Bacilli in ileal all increased compared with which before OF (Table 2) $(p<0.05)$.

The addition of HSCAS triggered an increase in representation of the class Bacilli from $26.97 \%$ without HSCAS to $69.02 \%$ with it. This increase coincided with a decrease in Actinobacteria from $22.58 \%$ to $9.26 \%$, Gammaproteobacteria from $10.22 \%$ to $6.34 \%$, Alphaproteobacteria from $12.64 \%$ to $4.39 \%$, and Clostridia from $4.43 \%$ to $3.17 \%$.

As for the effect of HSCAS on bacterial genera, Corynebacterium decreased at the end of OF without HSCAS, and increased dramatically when HSCAS was administered (Table 2). A statistically significant increase in total bacterial load and Firmicutes representative, 
Table 2 - Statistical effect of overfeeding and addition of HSCAS on different genera in the ileum or ceca of Landes geese.

\begin{tabular}{lcccc}
\hline \multirow{2}{*}{ Item } & \multicolumn{3}{c}{ Group } \\
\cline { 2 - 5 } lleum & Control & OF Control & HSCAS & \\
\hline Lactobacillus & $6.77 \pm 0.85^{\mathrm{c}}$ & $24.01 \pm 3.42^{\mathrm{b}}$ & $54.92 \pm 5.36^{\mathrm{a}}$ & 0.015 \\
Corynebacterium & $4.19 \pm 1.14^{\mathrm{b}}$ & $14.65 \pm 3.18^{\mathrm{a}}$ & $5.29 \pm 1.10^{\mathrm{b}}$ & 0.021 \\
Helicobacter & $3.39 \pm 1.22^{\mathrm{a}}$ & $0.82 \pm 0.31^{\mathrm{b}}$ & $0.22 \pm 0.09^{\mathrm{b}}$ & 0.048 \\
Actinobacillus & $0.50 \pm 0.23^{\mathrm{b}}$ & $5.42 \pm 1.06^{\mathrm{a}}$ & $2.54 \pm 1.52^{\mathrm{a}}$ & 0.048 \\
Pseudoalteromonas & $0.85 \pm 0.43$ & $0.03 \pm 0.01$ & $0.03 \pm 0.01$ & 0.069 \\
\hline Ceca & & & $0.01 \pm 1.54^{\mathrm{b}}$ & 0.001 \\
\hline Bacteroides & $30.26 \pm 2.13^{\mathrm{a}}$ & $11.09 \pm 3.31^{\mathrm{b}}$ & $0.12 \pm 0.02$ & 0.078 \\
Clostridium & $4.68 \pm 2.24$ & $0.22 \pm 0.10$ & $0.23 \pm 0.02$ & 0.075 \\
Desulfovibrio & $3.46 \pm 1.62$ & $0.12 \pm 0.03$ & $0.32 \pm 0.24^{\mathrm{b}}$ & 0.041 \\
Lactobacillus & $1.98 \pm 0.68^{\mathrm{a}}$ & $0.18 \pm 0.08^{\mathrm{b}}$ & $0.18 \pm 2.69^{\mathrm{b}}$ & 0.022 \\
Escherichia & $0.74 \pm 0.30^{\mathrm{b}}$ & $18.86 \pm 5.14^{\mathrm{a}}$ & & \\
\hline
\end{tabular}

$a, b:$ Mean values within a row with no common superscript differ significantly $(p<0.05)$.

including Lactobacillus, was observed. Helicobacter and Actinobacillus in the phylum Proteobacteria decreased substantially (Table 2).

\section{Effect of HSCAS on cecal microbial com- munities in overfed geese}

Before OF, the cecal microbiota consisted essentially of Bacteroidia (38.4\%) and Clostridia (34.4\%). Some changes were detected during OF, but these two classes remained dominant during OF. After OF, there was a significant increase in Gammaproteobacteria $(21.5 \%)$, concomitant with a decrease in Bacteroidia (19.2\%) and Clostridia (29.4\%). These three classes were dominant at the end OF, representing $70 \%$ of the total bacterial population (Figure 3b).

Treatment with HSCAS triggered an increase in the Deltaproteobacteria class from $0.9 \%$ without HSCAS to $31.3 \%$ with it. This increase coincided with a decrease in Gammaproteobacteria from 21.5\% to $6.9 \%$, whereas Clostridia decreased only slightly from 29.4 to $26.9 \%$ and Bacteroidia increased from $19.2 \%$ to $25.7 \%$. Treatment with HSCAS did not affect the number of Bacilli sequences in ceca. At the end of $\mathrm{OF}$, only a small percentage of Bacilli sequences (3.55\%) were found in the HSCAS group. In contrast, Erysipelotrichi sequences decreased from 14.60\% in HSCAS-free overfed birds to $1.65 \%$ in HSCAS-fed birds (Figure 3b).

Lactobacillus increased significantly at the end OF with HSCAS. A statistically significant decrease in the total bacterial load and representatives of the Firmicutes, including Clostridium, was observed. Sequences representing Bacteroides and Escherichia also decreased markedly (Table 2 ).

\section{DISCUSSION}

The liver is the main organ responsible for de novo lipogenesis in avian species (Li et al., 2015). Moreover, the liver is both the main organ for detoxification and the principal toxic target organ (Neeff et al., 2013). The best grade fatty liver is produced in France, where government regulations require stricter quality controls in foie gras production (Skippon, 2013; Gourmetfoodstore, 2016). For the sake of safety and quality of foie gras as well as good health of birds, the adverse effects should be avoided in the management of geese liver production.

Aluminosilicates (mainly zeolites, HSCAS) are known as feed additives used for multiple purposes in the poultry industry due to their capabilities as mycotoxin adsorbents and other qualifications. The histopathology of liver showed that lesions attributed to aflatoxicosis were ameliorated by adding 0.2\% HSCAS to the contaminated foods (Zhao et al., 2010). This substance binds to AF molecules in the gastrointestinal tract, making them unavailable for adsorption and consequently alleviating aflatoxicosis (Liu et al., 2011).

In the present study, overfeeding led to an increase in relative weight of liver compared with the negative control $(p<0.05)$. Overfeeding of HSCAS-treated geese exhibited benefit effects on fat deposition compared to those of overfeeding alone, with the liver percentage relative to body weight increasing $2.50 \%(p<0.05)$ at the end of the overfeeding period. Su et al., (2009) investigated the impact of betaine supplementation on the fatty livers in geese and found that the betaine diet increased liver weight by more than $100 \mathrm{~g}$ (Su et al., 2009). Betaine has been considered an ideal hepatic protectant against both alcoholic (Ji \& Kaplowitz, 2003; 
Day \& Kempson, 2016) and non-alcoholic steatosis (Neuschwander-Tetri, 2001; Chen et al., 2015).

With respect to HSCAS, it has reduced the incidence and severity of the hepatic histopathology changes associated with aflatoxicosis (Chen et al., 2014). In the present study, HSCAS supplementation increased the relative liver weight. This suggests that lipogenesis and fatty liver production was thus improved in the HSCAStreated geese. The HSCAS, which induces beneficial effects on the apparent nutrient retention, may be associated with decreasing lesions in gastrointestinal tract through the effective absorption of aflatoxins (Liu et al., 2011). This may explain the improved liver weight and liver to body weight. A healthy gut not only affects nutrient utilization in the birds, but also has exerts considerable influence on the microbiota composition of the bird, which was observed in the ileal and cecal microbiota of geese in this study.

In other studies on the intestinal microbiota of birds and mammals, Firmicutes and Bacteroidetes were identified as the major phyla, suggesting their importance in metabolism and host physiology (Kohl, 2012). In contrast, the current study showed Firmicutes and Proteobacteria to be the major phyla. Firmicutes was the second most common phylum after Proteobacteria in ileal samples, based on HiSeq2500 sequencing. Firmicutes was also the major phylum in feces of Canada geese (Lu et al., 2009) and Muscovy ducks (Cairina moschata)(Vasai et al., 2014a), whereas Bacteroidetes are less abundant in some avian species (Lu et al., 2007). In contrast to mule ducks, where obligate anaerobes (class Clostridia and Bacteroidia) dominated in both ileal and cecal samples (Vasai et al., 2014b), in geese, obligate anaerobes (class Clostridia of Firmicutes and Bacteroidetes) dominated the ceca samples, and facultative anaerobes (Gammaproteobacteria, Bacilli, and especially Lactobacillus) were dominant in the ileum. Higher numbers Clostridia sequences were observed in ducks than in geese, suggesting that bacterial digestive metabolism in geese and ducks may be somewhat different.

OF increased the relative abundance of Firmicutes, and especially Lactobacillus, in ileal samples from geese, as previously described in mule, Pekin, and Muscovy ducks (Vasai et al., 2014a; Vasai et al., 2014b). The Lactobacillus group, members of the class Bacilli, are known as amylolytic bacteria and frequently increase in pigs fed with diets rich in starch (Regmi et al., 2011). According to the high-throughput sequencing results, Lactobacillus, Actinobacteria, and Corynebacterium numbers increased; however, for Corynebacterium and
Actinobacteria, this increase was reversed by HSCAS supplementation. Helicobacter, in contrast, decreased with HSCAS supplementation.

HSCAS increased the diversity indexes of samples from the ileum, whereas in the ceca, an increase in these indexes only occurred in comparison with control II, while they decreased relative to control I. In broiler chicks, the addition of $0.5 \%$ HSCAS to an aflatoxin B1 diet significantly improved feed intake and weight gain (Gowda et al., 2008). In the current study, the weight of the fatty liver, as well Firmicutes/Bacteroides ratios, increased in comparison with those of overfed controls, but no significant effect on growth performance was observed (data not shown).

Feeding with HSCAS triggered an increase in ileal Bacilli numbers and an increase in sequences representing the phylum Firmicutes. The dominant sequences similar to those of Bacilli were most abundant in waterfowl, Canada geese, and gulls. On average, sequences similar to those of Bacteroidetes represent a smaller fraction of the avian fecal community, particularly in waterfowl (7.1\%) (Lu et al., 2009).

Thus far, there is little information on the effect of HSCAS on the diversity of goose fecal microbiota, although it has been hypothesized that HSCAS has a health impact on waterfowl gut microbial communities. In aquatic species, dietary supplementation with Azomite (an HSCAS product) enhances innate immunity and disease resistance in Oreochromis mossambicus against Aeromonas hydrophila (Musthafa et al., 2016).

In our study, the HSCAS diet resulted in an increased proportion of $16 \mathrm{~S}$ rRNA sequences in the Lactobacillus group, whereas the percentages of Proteobacteria, including Corynebacterium, Helicobacter and Altererythrobacter decreased. The relative abundance of Lactobacillaceae increased substantially, leading to a decrease in most other families (Vasai et al., 2014a). Previous studies showed that dietary supplementation with Lactobacillus-based probiotics increased gastrointestinal lactobacilli counts and decreased coliforms numbers (Hassan \& Ryu, 2012). Vasaï reported that the addition of Lactobacillus sakei triggers major changes in the ileum, whereas ceca were not affected. Lactobacillus sakei decreased the relative abundance of Bacteroides at mid-OF and the relative abundance of Enterobacteria in the ileum at the end of OF (Vasai et al., 2014b). The above-mentioned results are in agreement with a study in mice, in which probiotic treatment ameliorated metabolic syndrome symptoms, as well as increased Lactobacillus spp. and Bifidobacterium spp. and decreased Clostridiaceae 
spp., Akkermansia spp., and Escherichia coli (Park et al., 2013).

The decrease in these potentially pathogenic bacteria was simultaneous with an increase in Bifidobacterium spp. and Lactobacillus spp./Enterococcus spp. (Rawski etal., 2016). In poultry species, dietary supplementation with Lactobacillus-based probiotics suppressed the growth of potentially pathogenic bacteria such as Clostridium, Escherichia coli, and Salmonella enterica, as determined by culture-dependent methods (Vasai et al., 2014b). Supplementation with HSCAS resulted in a decrease in Helicobacter and Actinobacillus, suggesting that HSCAS can protect against potential pathogens. The number of sequences representing Corynebacterium also decreased significantly with HSCAS supplementation, suggesting some level of antagonism between HSCAS and these bacteria. Because a decrease in the total bacterial load in ileum was observed at the end of OF, it is possible that HSCAS inhibited growth of several other bacteria.

The cecal samples showed a very different pattern than the ileal samples in response to HSCAS. First, supplementation with HSCAS altered the microbial communities, as determined by the nonmetric multidimensional scaling analysis and bacterial composition. In chickens, Clostridia and Bacteroides (obligate anaerobes) were predominant in cecal samples, whereas Lactobacillus spp. (facultative anaerobes) was an important component of the ileal microbiota (Lu et al., 2003). A study of two parental genetic types (Pekin and Muscovy ducks) showed that cecal microbiota was mainly composed of Firmicutes and Bacteroidetes (Vasai et al., 2014a). Both Lactococcus and Paenibacillus spp. have been isolated from poultry ceca or feces, so they can be considered normal members of the avian gut microbiota (Lu et al., 2009). The complex anaerobic environment may be refractory to changes in the microbial community (Vasai et al., 2014b). In this study, HSCAS feeding had an effect in ceca. Treatment with HSCAS increased representation of the Deltaproteobacteria class from $0.9 \%$ without HSCAS to $31.3 \%$ with it. This increase coincided with a decrease in Gammaproteobacteria from 21.5\% to $6.9 \%$. OF led to a decrease in Bacteroides, Clostridium and Escherichia numbers at the end of OF; however, for Clostridium and Escherichia, this decrease was reversed by HSCAS supplementation.

In addition, we observed an increase of the Erysipelotrichi class after OF in Landes geese, which was reduced by HSCAS treatment. Previously, the class Erysipelotrichi was reported to be overabundant in the obese state (Turnbaugh et al., 2009). Overgrowth of members of the class Erysipelotrichi was observed after feeding obese mice but not lean mice after feeding with a high-fat diet (Upadhyay et al., 2012). This implicated Erysipelotrichi as a species that might contributed to metabolic disease in a host on a highfat diet (Upadhyay et al., 2012); however, overgrowth of this class of bacteria was reversed by HSCAS in the current study. Thus, HSCAS may have the beneficial effect of restoring balance to gut microbes.

Bacteroides are predominant in intestines because of their ability to utilize polysaccharides (Ravcheev et al., 2013). An imbalance in the gut microbiota, known as dysbiosis, is usually associated with a sudden increase in the abundance of facultative anaerobic Gammaproteobacteria, particularly Enterobacteriaceae, which is characteristic of gut malfunction and intestinal inflammation (Winter et al., 2013). HSCAS reduced the number of Gammaproteobacteria and increased that of Bacteroidia. HSCAS can be inferred to play a role in reducing inflammation. In the Proteobacteria, the class Gammaproteobacteria has been shown to increase its numbers with increasing cholic acid concentrations (Islam et al., 2011). The relative abundance of members of the order Enterobacteriales (class Gammaproteobacteria) have been reported to significantly increase in rats fed a high-fat diet, and Enterobacteriaceae are known to be highly tolerant of bile acid (de La Serre et al., 2010). A high-fat diet enhances bile secretion to facilitate lipid digestion (Reddy, 1981). Cholic acid feeding simplified the composition of the microbiota, with the overgrowth of several bacteria in the classes Clostridia and Erysipelotrichi (Islam et al., 2011). This effect may explain the increase in Gammaproteobacteria and decrease in Bacteroidia in the ceca of overfed geese.

\section{CONCLUSION}

In conclusion, dietary HSCAS supplementation promoted liver growth performance in overfed Landes geese. This study, using high-throughput sequencing based on 16S rRNA in ileal and cecal samples, provides information on the microbial communities in Landes geese and the effect of administering HSCAS on the abundance of major groups during OF. The gut microbial community in geese is dominated by Firmicutes, Bacteroidetes, and Proteobacteria, and OF modifies the bacterial communities primarily in cecal samples, whereas HSCAS shows an important effect on ileal samples. The increase in Lactobacillus during 
OF with the addition of HSCAS as a prebiotic, may improve the bird's health and therefore be of great interest to the poultry industry.

\section{ACKNOWLEDGMENTS}

The authors thank Beijing Novogene Biotechnology Co. Ltd. for performing high-throughput sequencing and data analysis. This project was supported by the International Science \& Technology Cooperation Program of China (2013DFA31880) and Zhejiang Major Scientific and Technological Project of Agricultural (Livestock) Breeding (2012C12906-14).

\section{CONFLICT OF INTEREST}

All authors read and approved the findings of the study. None of the authors have any conflicts of interest. We certify that there is no conflict of interest with any financial organization regarding the material discussed in the manuscript.

\section{REFERENCES}

Aravind KL, Patil VS, Devegowda G, Umakantha B, Ganpule SP. Efficacy of esterified glucomannan to counteract mycotoxicosis in naturally contaminated feed on performance and serum biochemical and hematological parameters in broilers. Poultry Science 2003;82(4):571576.

Bokulich NA, Subramanian S, Faith JJ, Gevers D, Gordon JI, Knight R, et al. Quality-filtering vastly improves diversity estimates from Illumina amplicon sequencing. Nature Methods 2013;10(1):57-59.

Caporaso JG, Kuczynski J, Stombaugh J, Bittinger K, Bushman FD, Costello EK, et al. QIIME allows analysis of high-throughput community sequencing data. Nature Methods 2010;7(5):335-336.

Chen J, Zhou X, Wu W, Wang X, Wang Y. FTO-dependent function of N6-methyladenosine is involved in the hepatoprotective effects of betaine on adolescent mice. Journal of Physiology and Biochemistry 2015;71(3):405-413

Chen X, Horn N, Applegate TJ. Efficiency of hydrated sodium calcium aluminosilicate to ameliorate the adverse effects of graded levels of aflatoxin B1 in broiler chicks. Poultry Science 2014;93(8):2037-2047.

Davail S, Rideau N, Guy G, Andre JM, Hoo-Paris R. Pancreatic hormonal and metabolic responses in overfed ducks. Hormone and Metabolic Research 2003;35(7):439-443

Day CR, Kempson SA. Betaine chemistry, roles, and potential use in liver disease. Biochimica Biophysica Acta 2016;1860(6):1098-1106.

De La Serre CB, Ellis CL, Lee J, Hartman AL, Rutledge JC, Raybould HE. Propensity to high-fat diet-induced obesity in rats is associated with changes in the gut microbiota and gut inflammation. American Journal of Physiology-Gastrointestinal Liver Physiology 2010;299(2):G440-G448.

Du X, Xu XQ, Tian Y, Shen JL, Lu LZ, Niu D. Morphology and microbiota of different section of intestines and their influences induced by dietary fish oil in Shan Partridge Ducks (Anas platyrhynchos). Journal of Agricultural Biotechnology 2016;11(24):11-24.
Gilani A, Kermanshahi H, Golian A, Seifi S. Appraisal of the impact of aluminosilicate use on the health and performance of poultry. Turkish Journal of Veterinary and Animal Science 2016;40(3):255-262.

GourmetFoodStore. Grades of foie gras. 2016. Available from: https:// www.gourmetfoodstore.com/grades-foie-gras-15164.

Gowda NK, Ledoux DR, Rottinghaus GE, Bermudez AJ, Chen YC. Efficacy of turmeric (Curcuma longa), containing a known level of curcumin, and a hydrated sodium calcium aluminosilicate to ameliorate the adverse effects of aflatoxin in broiler. Poultry Science 2008;87(6):1125-1130.

Haas BJ, Gevers D, Earl AM, Feldgarden M, Ward DV, Giannoukos G, et al. Chimeric 16S rRNA sequence formation and detection in Sanger and 454-pyrosequenced PCR amplicons. Genome Research 2011;21(3):494-504.

Hassan MR, Ryu KS. Naturally derived probiotic supplementation effects on physiological properties and manure gas emission of broiler chickens. Journal of Agriculture \& Life Sciences 2012;4(46):119-127.

Islam KBMS, Fukiya S, Hagio M, Fujii N, Ishizuka S, Ooka T, et al. Bile acid is a host factor that regulates the composition of the cecal microbiota in rats. Gastroenterology 2011;141(5):1773-1781.

Ji C, Kaplowitz N. Betaine decreases hyperhomocysteinemia, endoplasmic reticulum stress, and liver injury in alcohol-fed mice. Gastroenterology 2003;124(5):1488-1499.

Kohl KD. Diversity and function of the avian gut microbiota. Journal of Comparative Physiology B 2012;182(5):591-602.

Leser TD, Molbak L. Better living through microbial action: the benefits of the mammalian gastrointestinal microbiota on the host. Environmental Microbiology 2009;11(9):2194-2206

Li H, Wang T, Xu C, Wang D, Ren J, Li Y, et al. Transcriptome profile of liver at different physiological stages reveals potential mode for lipid metabolism in laying hens. BMC Genomics 2015;16:763.

Liu L, Zhao X, Wang Q, Sun X, Xia L, Wang Q, Yang B, Zhang Y, Montgomery $S$, Meng H, Geng T, Gong D. Prosteatotic and protective components in a unique model of fatty liver: gut microbiota and suppressed complement system. Scientific Reports 2016;6(1):31763.

Liu YL, Meng GQ, Wang HR, Zhu HL, Hou YQ, Wang WJ, et al. Effect of three mycotoxin adsorbents on growth performance, nutrient retention and meat quality in broilers fed on mould-contaminated feed. British Poultry Science 2011;52(2):255-263.

Lu J, Idris U, Harmon B, Hofacre C, Maurer JJ, Lee MD. Diversity and succession of the intestinal bacterial community of the maturing broiler chicken. Applied Environmental Microbiology 2003;69(11):6816-6824.

Lu J, Santo Domingo J, Shanks OC. Identification of chicken-specific fecal microbial sequences using a metagenomic approach. Water Research 2007;41(16):3561-3574.

Lu J, Santo Domingo JW, Hill S, Edge TA. Microbial diversity and hostspecific sequences of Canada goose feces. Applied Environmental Microbogy 2009;75(18):5919-5926.

Lu L, Chen Y, Wang Z, Li X, Chen W, Tao Z, et al. The goose genome sequence leads to insights into the evolution of waterfowl and susceptibility to fatty liver. Genome Biology 2015;16(1):89.

Murugesan GR, Ledoux DR, Naehrer K, Berthiller F, Applegate TJ, Grenier $B$, et al. Prevalence and effects of mycotoxins on poultry health and performance, and recent development in mycotoxin counteracting strategies. Poultry Science 2015;94(6):1298-1315.

Musthafa MS, Ali ARJ, Mohamed MJ, Jaleel MMA, Kumar MSA, Rani KU, et al. Protective efficacy of Azomite enriched diet in Oreochromis 
Tang J, Fang Q, Lu M, Shao R,

Shen J, Lu L, Niu D
The Effect of Hydrated Sodium Calcium

Aluminosilicate on Fatty Liver and the Composition of the Intestinal Microbiota in Overfed Landes Geese mossambicus against Aeromonas hydrophila. Aquaculture 2016;451(Suppl C):310-315.

Neeff DV, Ledoux DR, Rottinghaus GE, Bermudez AJ, Dakovic A, Murarolli RA, et al. In vitro and in vivo efficacy of a hydrated sodium calcium aluminosilicate to bind and reduce aflatoxin residues in tissues of broiler chicks fed aflatoxin B1. Poultry Science 2013;92(1):131-137.

Neuschwander-Tetri BA. Betaine: an old therapy for a new scourge. American Journal of Gastroenterology 2001;96(9):2534-2536.

Nylund L, Heilig HGHJ, Salminen S, de Vos WM, Satokari R. Semiautomated extraction of microbial DNA from feces for $\mathrm{qPCR}$ and phylogenetic microarray analysis. Journal of Microbiological Methods 2010;83(2):231-235

Park DY, Ahn YT, Park SH, Huh CS, Yoo SR, Yu R, et al. Supplementation of Lactobacillus curvatus HY7601 and Lactobacillus plantarum KY1032 in diet-induced obese mice is associated with gut microbial changes and reduction in obesity. PLoS One 2013;8(3):e59470.

Ravcheev DA, Godzik A, Osterman AL, Rodionov DA. Polysaccharides utilization in human gut bacterium Bacteroides thetaiotaomicron: comparative genomics reconstruction of metabolic and regulatory networks. BMC Genomics 2013;14:873.

Rawski M, Kieronczyk B, Dlugosz J, Swiatkiewicz S, Jozefiak D. Dietary probiotics affect gastrointestinal microbiota, histological structure and shell mineralization in turtles. PLoS One 2016;11(2):e147859.

Reddy BS. Diet and excretion of bile acids. Cancer Research 1981;41(9 Pt 2):3766-3768

Regmi PR, Metzler-Zebeli BU, Ganzle MG, van Kempen TA, Zijlstra RT. Starch with high amylose content and low in vitro digestibility increases intestinal nutrient flow and microbial fermentation and selectively promotes bifidobacteria in pigs. Journal of Nutrition 2011;141(7):12731280.

Salim HM, Kang HK, Akter N, Kim DW, Kim JH, Kim MJ, et al. Supplementation of direct-fed microbials as an alternative to antibiotic on growth performance, immune response, cecal microbial population, and ileal morphology of broiler chickens. Poultry Science 2013:92(8):2084-2090.

Serino M, Luche E, Gres S, Baylac A, Bergé M, Cenac C, et al. Metabolic adaptation to a high-fat diet is associated with a change in the gut microbiota. Gut 2012;61(4):543-553.

Shang QH, Yang ZB, Yang WR, Li Z, Zhang GG, Jiang SZ. Toxicity of mycotoxins from contaminated corn with or without yeast cell wall adsorbent on broiler chickens. Asian-Australasian Journal of Animal Science 2016;29(5):674-680.

Skippon W. The animal health and welfare consequences of foie gras production. Canadian Veterinary Journal 2013;54(4):403-404.

SPSS. IBM SPSS statistics 20 core. System user's guide. Chicago; 2011.
Su SY, Dodson MV, Li XB, Li QF, Wang HW, Xie Z. The effects of dietary betaine supplementation on fatty liver performance, serum parameters, histological changes, methylation status and the mRNA expression level of Spot14 $\alpha$ in Landes goose fatty liver. Comparative Biochemistry and Physiology Part A: Molecular \& Integrative Physiology 2009;154(3):308314.

Swamy HV, Smith TK, Cotter PF, Boermans HJ, Sefton AE. Effects of feeding blends of grains naturally contaminated with Fusarium mycotoxins on production and metabolism in broilers. Poultry Science 2002;81(7):966975.

Tanpong S, Wongtangtintharn S, Pimpukdee K, Tengjaroenkul B, Khajarern J. Efficacy of hydrate sodium calcium aluminosilicate and yeast cell wall to ameliorate the toxic effects of aflatoxin in ducks. Animal Production Science 2017;57(8):1637-1644.

Torok VA, Hughes RJ, Mikkelsen LL, Perez-Maldonado R, Balding K, MacAlpine $R$, et al. Identification and characterization of potential performance-related gut microbiotas in broiler chickens across various feeding trials. Applied Environmental Microbiology 2011;77(17):58685878.

Turnbaugh PJ, Ridaura VK, Faith JJ, Rey FE, Knight R, Gordon Jl. The effect of diet on the human gut microbiome: a metagenomic analysis in humanized gnotobiotic mice. Science Translational Medicine 2009;1(6):6r-14r.

Upadhyay V, Poroyko V, Kim TJ, Devkota S, Fu S, Liu D, et al. Lymphotoxin regulates commensal responses to enable diet-induced obesity. Nature Immunology 2012;13(10):947-953.

Vasai F, Brugirard RK, Bernadet MD, Cauquil L, Bouchez O, Combes S, et al. Overfeeding and genetics affect the composition of intestinal microbiota in Anas platyrhynchos (Pekin) and Cairina moschata (Muscovy) ducks. FEMS Microbiology Ecology 2014a;87(1):204-216.

Vasai F, Ricaud KB, Cauquil L, Daniel P, Peillod C, Gontier K, et al. Lactobacillus sakei modulates mule duck microbiota in ileum and ceca during overfeeding. Poultry Science 2014b;93(4):916-925.

Winter SE, Winter MG, Xavier MN, Thiennimitr P, Poon V, Keestra AM, et al. Host-derived nitrate boosts growth of $\mathrm{E}$. coli in the inflamed gut. Science 2013;339(6120):708-711.

Xie Q, Pan M, Huang R, Tian X, Tao X, Shah NP, et al. Short communication: Modulation of the small intestinal microbial community composition over short-term or long-term administration with Lactobacillus plantarum ZDY2013. Journal of Dairy Science 2016;99(9):6913-6921.

Zhao J, Shirley RB, Dibner JD, Uraizee F, Officer M, Kitchell M, et al. Comparison of hydrated sodium calcium aluminosilicate and yeast cell wall on counteracting aflatoxicosis in broiler chicks. Poultry Science 2010;89(10):2147-2156. 